\title{
ON NONBLOCKING MULTICONNECTION NETWORKS COMPOSED OF DIGITAL SWITCHING MATRICES
}

\author{
ANDRZEJ JAJSZCZYK ${ }^{1}$
}

(Received November 1988)

\begin{abstract}
In the paper we study the conditions under which multiconnection networks are nonblocking. A multiconnection network deals with the connections of pairs $\left\{\left(T_{1}, T_{2}\right)\right\}$ where $T_{1}$ is a subset of the input terminals and $T_{2}$ is a subset of the output terminals. We investigate networks composed of digital switching matrices. Such networks can be treated as a very general case encompassing many kinds of networks used in practice as well as studied theoretically.

We present four routing strategies and then develop conditions under which multiconnection networks are nonblocking when each of these strategies is used. We also show that the obtained conditions reduce to known results for some values of network parameters.
\end{abstract}

\section{Introduction}

A multiconnection network deals with the connections of pairs $\left\{\left(T_{1}, T_{2}\right)\right\}$ where $T_{1}$ is a subset of the input terminals and $T_{2}$ is a subset of the output terminals [5]. Multiconnections are often used in practice, for example, for conference connections, for loop connection tests, or for connections of several subscribers to a single service circuit, such as a signal sender. A special case of multiconnection networks are broadcast networks, in which $\left|T_{1}\right|=1$. Recently the special interest in multiconnection networks is caused by their application in broadband systems used for the transmission and switching of high bit-rate signals, including $T V$ and high speed data [14], [15].

Space-division multiconnection networks have been dealt with by several authors. Hwang and Jajszczyk have given the conditions under which such networks are nonblocking [4], [5], [6]. The results obtained for space-division

\footnotetext{
${ }^{1}$ Inst. of Elec. \& Comm., Tech. University of Poznań, ul. Piotrowo 3a, 60-965 Poznań, Poland. (C) Copyright Australian Mathematical Society 1989, Serial-fee code 0334-2700/89
} 


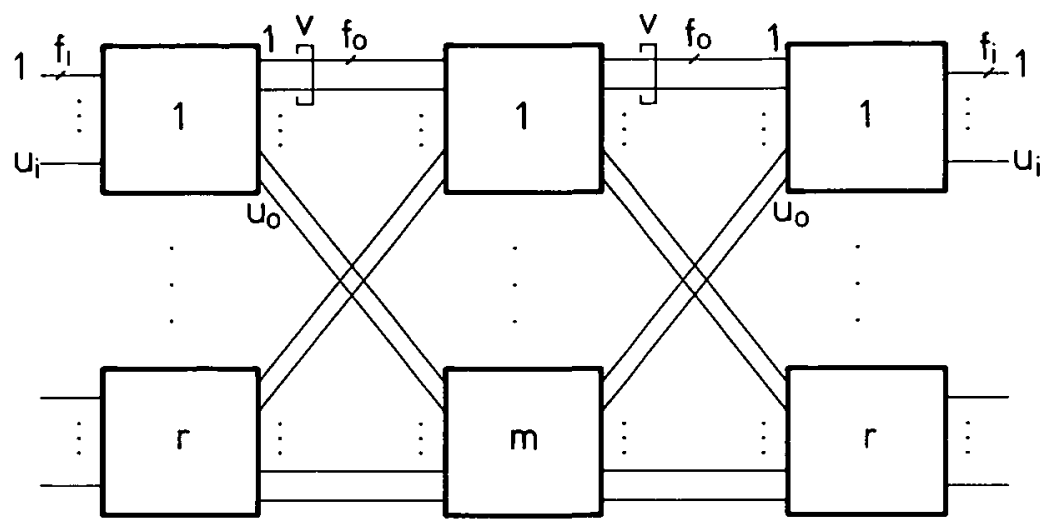

FIGURE 1. Three-stage network; $u$ : number of links, $v$ : number of links between the same switches, $f$ : number of channels in a link.

networks are applicable to time-division networks having separate time and space stages. However, they cannot be applied to networks composed of digital switching matrices, the introduction of which is changing design concepts for digital switching networks.

Digital switching matrix (DSM) mix in the same chip, or in the same printed circuit board, time and space switching [1]. In the matrix information can be transferred from any channel of any incoming PCM link to any channel of any outgoing PCM link. The structures and some features of various switching networks composed of such elements have been discussed in several papers [2], [7], [9], [13], [16].

In this paper the conditions under which multiconnection networks composed of DSMs are nonblocking will be formulated and proven. To illustrate the principle we shall consider the three-stage network shown in Fig. 1. The structure is similar to that proposed by Clos [3]. The main difference is the multiple linkage connection between pairs of switches in successive stages (this multiple linkage connection will be referred to as a "bundle of links") and that each link can carry more than one channel. The analysis of such a network resolves itself into the analysis of a multiple linkage space-division network.

Let $I$ denote the set of input terminals (input channels) and $O$ the set of output terminals (output channels). A connecting pair in a multiconnection network is defined to be a pair $\left(T_{1}, T_{2}\right): T_{1} \subseteq I, T_{2} \subseteq O$ such that each terminal in $T_{1}$ is to be connected to every terminal in $T_{2}$. In a $\left(q_{1}, q_{2}\right)$-multiconnection network all connecting pairs must satisfy the restrictions $\left|T_{1}\right| \leq q_{1}$ and $\left|T_{2}\right| \leq$ $q_{2}$. A multiconnection network is nonblocking if, regardless of what state the network is currently in, a connecting pair involving only idle terminals can 
always be connected by a subgraph of the network which is link-disjoint from all subgraphs connecting previous pairs.

Throughout this paper it is assumed that each rectangular switch has the fan-in, fan-out property, i.e., any subset of inlets can be connected simultaneously to any subset of outlets. The above assumption is, of course, valid for space-division switches. However, it cannot be used for some kinds of digital switching matrices. For example, in the DSM described by Belforte et al. the address memory controls the output of the speech memory, and therefore we can send a speech sample from any channel of any incoming PCM link to a multiplicity of outgoing channels, but not vice versa [1]. Some structures of digital switches having both sides of speech memory controlled have been reported [12]. For the sake of generality, we shall use the "fan-in, fan-out" assumption. Therefore, we can restrict ourselves to consider pairs $\left(T_{1}, T_{2}\right)$ consisting of at most one terminal from each input switch and at most one terminal from each output switch, because if we can connect one input (output) terminal to $T_{2}\left(T_{1}\right)$, then all terminals in the same input (output) switch can be connected to $T_{2}\left(T_{1}\right)$. Hence, we may assume $r \geq q_{1}$ and $r \geq q_{2}$ without a loss of generality. We also assume that overlapping of two or more connecting pairs is not allowed.

Throughout the paper by $\lfloor x\rfloor$ we denote the greatest integer less than or equal to $x$, and by $\lceil x\rceil$ we denote the smallest integer greater than or equal to $x$.

\section{Routing strategies}

We shall modify the four routing strategies proposed by Hwang in order to use them in the discussed networks. The strategies can be formulated as follows.

Strategy 1. A connecting path for each pair $\left(t_{1}, t_{2}\right), t_{1} \in T_{1}, t_{2} \in T_{2}$ is set up independently of a path for another pair $\left(t_{1}, t_{2}\right)$. By "independence" we shall mean that any channel is not used by more than one pair $\left(t_{1}, t_{2}\right)$.

Strategy 2. A connecting path for each pair $\left(t_{1}, T_{2}\right), t_{1} \in T_{1}$ is set up through a single channel leading from the input stage to a middle-stage switch. Then it is branched out in this switch and leads to all $t_{2} \in T_{2}$.

Strategy 3. A connecting path for each pair $\left(T_{1}, t_{2}\right), t_{2} \in T_{2}$ is set up through a single channel connecting the output and the middle stages. Then it is branched out in the middle-stage switch and leads to all $t_{1} \in T_{1}$. 
STRATEgy 4. A connecting path for each pair $\left(T_{1}, T_{2}\right)$ is set up through a single middle-stage switch; all elements $t_{1}$ and $t_{2}, t_{1} \in T_{1}, t_{2} \in T_{2}$ are connected to this switch through single channels.

It can be easily seen that for $v f_{o}=1$ the presented strategies reduce to the strategies described in [5].

\section{Conditions for nonblocking}

Consider the pair $\left(T_{1}, T_{2}\right)$. Let $t_{1} \in T_{1}, t_{2} \in T_{2}$ and let $S_{i}$ be the set of switches accommodating $T_{i}$ where $i=1,2$. Denote $y_{i}=\left|T_{i}\right|$. Because of the fan-in, fan-out properties, we may assume that $\left|T_{i}\right|=\left|S_{i}\right|=y_{i}$. Let $s_{i}$ be the switch accommodating $t_{i}$.

We can formulate the following four theorems.

THEOREM 1. A two-sided three-stage network composed of DSMs is nonblocking as a $\left(q_{1}, q_{2}\right)$-multiconnection network under Strategy 1 if and only if

$$
\begin{aligned}
m \geq 1+\min \left\{\left\lfloor\left(u_{i} f_{i} q_{1}-1\right) /\left(v f_{o}\right)\right\rfloor+\right. & \left\lfloor\left(u_{i} f_{i} q_{2}-1\right) /\left(v f_{o}\right)\right\rfloor, \\
& \left.\left\lfloor\left(u_{i} f_{i}\left(r+\min \left\{q_{1}, q_{2}\right\}-1\right)-1\right) /\left(v f_{o}\right)\right\rfloor\right\} .
\end{aligned}
$$

Proof. Sufficiency. Consider the connection of the pair $\left(T_{1}, T_{2}\right)$. Let $g_{1}$ be the maximum number of connections between the following sets of inputs and outputs $s_{1}-t_{1}$ and $O-\left\{s_{2} \cup T_{2}\right\}$. Let $g_{2}$ be the maximum number of connections between the sets $s_{2}-t_{2}$ and $I-\left\{s_{1} \cup T_{1}\right\}$. Let $g_{3}$ be the maximum number of connections between the sets $s_{1}-t_{1}$ and $s_{2}-t_{2}$. Let $g_{4}$ be the number of connections between $t_{1}$ and $T_{2}-t_{2}$. Let $g_{5}$ be the number of connections between $t_{2}$ and $T_{1}-t_{1}$. Let $g_{6}$ be the number of connections between $t_{1}$ and $t_{2}$.

Similarly as in the proof of Theorem 1 in [5], the numbers $g_{1}$ to $g_{6}$, for the worst state of a network, are as follows:

$$
\begin{aligned}
& g_{1}=\min \left\{\left(u_{i} f_{i}-1\right) \min \left\{q_{2}, r-1\right\},(r-1) u_{i} f_{i}-y_{2}+1\right\}, \\
& g_{2}=\min \left\{\left(u_{i} f_{i}-1\right) \min \left\{q_{1}, r-1\right\},(r-1) u_{i} f_{i}-y_{1}+1\right\}, \\
& g_{3}=\min \left\{u_{i} f_{i}-1,\left(u_{i} f_{i}-1\right) q_{2}-g_{1},\left(u_{i} f_{i}-1\right) q_{1}-g_{2}\right\}, \\
& g_{4}=y_{2}-1, \\
& g_{5}=y_{1}-1, \\
& g_{6}=1 .
\end{aligned}
$$

The total number of connections is then $g=g_{1}+g_{2}+g_{3}+g_{4}+g_{5}+g_{6}$. Since $g$ depends on $y_{1}$ and $y_{2}$ linearly, it is easily seen that $g$ achieves its 
maximum at $y_{1}=q_{1}$ and $y_{2}=q_{2}$. Hence,

$$
\left.g_{1}=\left(u_{i} f_{i}-1\right) \min \left\{q_{2}, r-1\right\}, \quad g_{2}=\left(u_{i} f_{i}-1\right) \min \left\{q_{1}, r-1\right)\right\} .
$$

Define $g^{0}=g\left(q_{1}, q_{2}\right)$. We consider the following four cases.

CASE 1. $q_{1}<r, q_{2}<r$. Then

$$
\begin{array}{rlrl}
g_{1}^{0}=\left(u_{i} f_{i}-1\right) q_{2}, & & g_{2}^{0}=\left(u_{i} f_{i}-1\right) q_{1}, \quad g_{3}^{0}=0, \\
g_{4}^{0}=q_{2}-1, & g_{5}^{0}=q_{1}-1, \quad g_{6}^{0}=1 .
\end{array}
$$

The connections $s_{1}-t_{1}$ to $O-\left\{s_{2} \cup T_{2}\right\}$ and $t_{1}$ to $T_{2}-t_{2}$ fully occupy $\left\lfloor\left(g_{1}^{0}+g_{4}^{0}\right) /\left(v f_{o}\right)\right\rfloor$ links between the first and the middle stages. Similarly, the connections $s_{2}-t_{2}$ to $I=\left\{s_{1} \cup T_{1}\right\}$ and $t_{2}$ to $T_{1}-t_{1}$ fully occupy $\left\lfloor\left(g_{2}^{0}+g_{5}^{0}\right) /\left(v f_{o}\right)\right\rfloor$ links between the middle and the third stages. Thus, in the worst state, the number of required middle-stage switches is

$$
h=\left\lfloor\left(u_{i} f_{i} q_{1}-1\right) /\left(v f_{o}\right)\right\rfloor+\left\lfloor\left(u_{i} f_{i} q_{2}-1\right) /\left(v f_{o}\right)\right\rfloor+1 .
$$

CASE 2. $q_{1}=r, q_{2}<r$. Then

(1) Between sets $s_{1}-t_{1}$ and $O-\left\{s_{2} \cup T_{2}\right\}$, and the sets $t_{1}$ and $T_{2}-t_{2}$ can exist $u_{i} f_{i} q_{2}-1$ connections (see Case 1$) ; v f_{o}\left\lfloor\left(u_{i} f_{i} q_{2}-1\right) /\left(v f_{o}\right)\right\rfloor$ of these connections fully occupy bundles of links coming to $\left\lfloor\left(u_{i} f_{i} q_{2}-1\right) /\left(v f_{o}\right)\right\rfloor$ middle-stage switches.

(2) The number of connections which can be established between the sets $t_{2}$ and $T_{1}-t_{1}$ is equal to $r-1$. The number of elements of the set $I-\left\{s_{1} \cup T_{1}\right\}$ limits $g_{2}$ (i.e. the maximum number of connections between $s_{2}-t_{2}$ and $I-\left\{s_{1} \cup T_{1}\right\}$ to $(r-1)\left(u_{i} f_{i}-1\right)$. Because in (1) only $v f_{o}\left\lfloor\left(u_{i} f_{i} q_{2}-1\right) /\left(v f_{o}\right)\right\rfloor$ connections of the set $s_{1}-t_{1}$ are used, the following number of connections can be established between the sets $s_{2}-t_{2}$ and $s_{1}-t_{1}: \min \left\{u_{i} f_{i}-1, R\right\}$, where $R=\left(u_{i} f_{i} q_{2}-1\right) \bmod \left(v f_{o}\right)$. Thus, connections discussed here can fully occupy bundles of links coming to $\left\lfloor\left((r-1) u_{i} f_{i}+\min \left\{u_{i} f_{i}-1, R\right\}\right) /\left(v f_{o}\right)\right\rfloor$ middle-stage switches.

In the worst state the sets of middle-stage switches described in (1) and (2) are disjoint. In order to connect the pair $\left(t_{1}, t_{2}\right)$ an additional switch is required. Therefore, the total number of required middle-stage switches is

$$
h\left\lfloor\left(u_{i} f_{i} q_{2}-1\right) /\left(v f_{o}\right)\right\rfloor+\left\lfloor\left((r-1)\left(u_{i} f_{i}+\min \left\{u_{i} f_{i}-1, R\right\}\right) /\left(v f_{o}\right)\right\rfloor+1 .\right.
$$

Because for positive integers $a, b, c, d$ holds

$$
d\lfloor a / b\rfloor+\lfloor(c+d[(a) \bmod (b)]) / b\rfloor=\lfloor(a d+c) / b\rfloor
$$

and $(r-1) u_{i} f_{i}+u_{i} f_{i}-1=u_{i} f_{i} q_{1}-1$ (in Case 2 we have $q_{1}=r$ ), expression (3) can be rewritten as follows

$$
\begin{aligned}
h=\min \left\{\left\lfloor\left(u_{i} f_{i} q_{1}-1\right) /\left(v f_{o}\right)\right\rfloor+\right. & {\left[\left(u_{i} f_{i} q_{2}-1\right) /\left(v f_{o}\right)\right\rfloor, } \\
& {\left.\left[\left(u_{i} f_{i}\left(r+q_{2}-1\right)-1\right) /\left(v f_{o}\right)\right\rfloor\right\}+1 . }
\end{aligned}
$$


CASE 3. $q_{1}<r, q_{2}=r$. By symmetry to Case 2 we have

$$
\begin{aligned}
h=\min \left\{\left\lfloor\left(u_{i} f_{i} q_{1}-1\right) /\left(v f_{o}\right)\right\rfloor+\right. & \left\lfloor\left(u_{i} f_{i} q_{2}-1\right) /\left(v f_{o}\right)\right\rfloor, \\
& \left.\left\lfloor\left(u_{i} f_{i}\left(r+q_{1}-1\right)-1\right) /\left(v f_{o}\right)\right\rfloor\right\}+1 .
\end{aligned}
$$

CASE 4. $q_{1}=q_{2}=r$. Then

$$
g_{1}^{0}=g_{2}^{0}=\left(u_{i} f_{i}-1\right)(r-1), \quad g_{3}^{0}=u_{i} f_{i}-1
$$

Because $\left(u_{i} f_{i}-1\right)(r-1)+u_{i} f_{i}-1=q_{j}\left(u_{i} f_{i}-1\right)$, for $j=1,2$, Case 4 is identical to Cases 2 and 3.

Summarising Cases 1-4 we obtain Theorem 1 . Necessity can be proved with ease by constructing sequences of calls which lead to the occupancy of such a number of switches which is given by formula (1).

Theorem 1 is illustrated by the following simple example.

EXAMPLE 1. Let us consider a network having the following parameters: $q_{1}=q_{2}=r=2, u_{i} f_{i}=5, v f_{o}=2$. By Theorem 1 the network is nonblocking for $m \geq 8$. The worst state of the network is presented in Figure 2 .

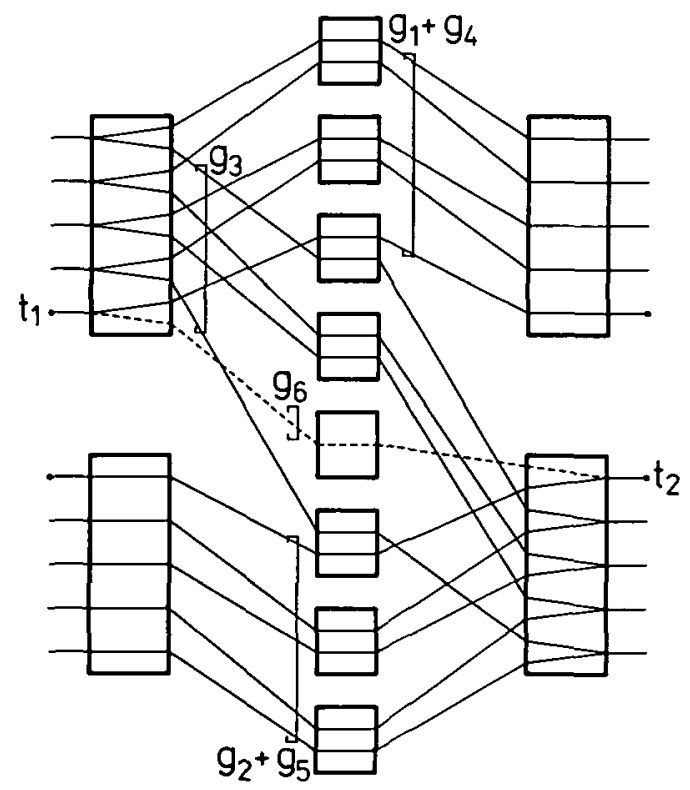

Figure 2. The worst state in the $(2,2)$-multiconnection network controlled by using Strategy 1 . 
THEOREM 2. A two-sided three-stage network composed of DSMs is nonblocking as $a\left(q_{1}, q_{2}\right)$-multiconnection network under Strategy 2 if and only if

$$
\begin{array}{r}
m \geq \max _{1 \leq y_{2} \leq q_{2}}\left\{\operatorname { m i n } \left\{\left\lfloor\left(u_{i} f_{i}-1\right) /\left(v f_{o}\right)\right\rfloor+y_{2}\left\lfloor\left(q_{1}\left(u_{i} f_{i}-1\right)\right) /\left(v f_{o}\right)\right\rfloor+\min \left\{d_{1}, d_{2}\right\},\right.\right. \\
\\
\left.\left.\left\lfloor\left(r u_{i} f_{i}-q_{1}\right) /\left(v f_{o}\right)\right\rfloor+d_{1},\left\lfloor\left(\left(r-y_{2}\right) u_{i} f_{i}+q_{1} y_{2}\left(u_{i} f_{i}-1\right)\right) /\left(v f_{o}\right)\right\rfloor+d_{2}\right\}\right\},
\end{array}
$$

where

$$
\begin{aligned}
& d_{1}=\left\{\begin{array}{l}
\left\lfloor\left(q_{1}-1\right) /\left(v f_{o}-R_{1}\right)\right\rfloor+1, \text { for } q_{1} \leq\left\lceil R_{2} / R_{1}\right\rceil v f_{o}-R_{2}, R_{1} \neq 0, \text { and } \\
\quad y_{2}\left\lfloor\left(q_{1}\left(u_{i} f_{i}-1\right)\right) /\left(v f_{o}\right)\right\rfloor \leq\left\lfloor\left(u_{i} f_{i}(r-1)-q_{1}+R+1\right) /\left(v f_{o}\right)\right\rfloor \\
\left\lfloor\left(q_{1}+R_{2}-1\right) /\left(v f_{o}\right)\right\rfloor+1 \text { for } q_{1}>\left\lceil R_{2} / R_{1}\right\rceil v f_{o}-R_{2}, R_{1} \neq 0 \\
\text { or } y_{2}\left\lfloor\left(q_{1}\left(u_{i} f_{i}-1\right)\right) /\left(v f_{o}\right)\right\rfloor>\left\lfloor\left(u_{i} f_{i}(r-1)-q_{1}+R+1\right) /\left(v f_{o}\right)\right\rfloor \\
\text { or } R_{1}=0
\end{array}\right. \\
& d_{2}=\left\{\begin{array}{l}
\left\lfloor\left(q_{1}-1\right)\left(v f_{o}-R_{1}\right)\right\rfloor+1, \text { for } q_{1} \leq\left\lceil R_{3} / R_{1}\right\rceil v f_{o}-R_{3}, R_{1} \neq 0 \\
\left\lfloor\left(q_{1}+R_{3}-1\right) /\left(v f_{o}\right)\right\rfloor+1, \quad \text { for } q_{1}>\left\lceil R_{3} / R_{1}\right\rceil v f_{o}-R_{3}, R_{1} \neq 0 \\
\left\lfloor\left(q_{1}-1\right)\left(v f_{o}\right)\right\rfloor+1, \text { for } R_{1}=0
\end{array}\right. \\
& R=\left(u_{i} f_{i}-1\right) \bmod \left(v f_{o}\right) \\
& R_{1}=\left(q_{1}\left(u_{i} f_{i}-1\right)\right) \bmod \left(v f_{o}\right) \text {; } \\
& R_{2}=u_{i} f_{i}(r-1)-q_{1}+1+R- \\
& -\min \left\{y_{2}\left\lfloor\left(q_{1}\left(u_{i} f_{i}-1\right)\right) /\left(v f_{o}\right)\right\rfloor,\left\lfloor\left(u_{i} f_{i}(r-1)-q_{1}+R+1\right) /\left(v f_{o}\right)\right\rfloor\right\} v f_{o} \text {; } \\
& R_{3}=\left\{y_{2} R_{1},\left(r-y_{2}\right) u_{i} f_{i}+y_{2} R_{1}-\right. \\
& \left.-\min \left\{\left\lfloor\left(u_{i} f_{i}-1\right) /\left(v f_{o}\right)\right\rfloor,\left\lfloor\left(\left(r-y_{2}\right) u_{i} f_{i}+y_{2} R_{1}\right) /\left(v f_{o}\right)\right\rfloor\right\} v f_{o}\right\} .
\end{aligned}
$$

Proof. Sufficiency. Consider the connection of the pair $\left(T_{1}, T_{2}\right)$. Let $g_{1}$ be the maximum number of connections between the sets $s_{1}-t_{1}$ and $O-S_{2}$. Let $g_{2}$ be the maximum number of connections between the sets $S_{2}-T_{2}$ and $I-\left\{s_{1} \cup T_{1}\right\}$. Let $g_{3}$ be the maximum number of connections between the sets $s_{1}-t_{1}$ and $S_{2}-T_{2}$. Let $g_{4}$ be the number of connections between the sets $T_{1}$ and $T_{2}$. It is easily seen that:

$$
\begin{aligned}
& g_{1}=\min \left\{u_{i} f_{i}-1,\left(r-y_{2}\right) u_{i} f_{i}\right\}, \\
& g_{2}=\min \left\{y_{2}\left(u_{i} f_{i}-1\right) \min \left\{q_{1}, r-1\right\},(r-1) u_{i} f_{i}-y_{1}+1\right\}, \\
& g_{3}=\min \left\{\left(u_{i} f_{i}-1\right) y_{2}, y_{2}\left(u_{i} f_{i}-1\right) q_{1}-g_{2}, u_{i} f_{i}-1-g_{1}\right\}, \\
& g_{4}=y_{1} .
\end{aligned}
$$

Because the total number of connections increases in $y_{1}$, so we can take $y_{1}=q_{1}$. Hence, $g_{2}$ can be rewritten as follows:

$$
g_{2}=\min \left\{y_{2}\left(u_{i} f_{i}-1\right) q_{1},(r-1) u_{i} f_{i}-q_{1}+1\right\} .
$$


The total number of connections is then $g=g_{1}+g_{2}+g_{3}+g_{4}$. Define $g^{0}=$ $g\left(q_{1}, y_{2}\right)$. We consider all possible combinations of the terms of $g_{1}, g_{2}, g_{3}$, and $g_{4}$ in $g^{0}$. There are 12 such combinations. We express a combination as follows:

$$
g_{1, i}+g_{2, j}+g_{3, k}+g_{4}
$$

where

$$
\begin{aligned}
g_{1,1} & =u_{i} f_{i}-1, \quad g_{1,2}=\left(r-y_{2}\right) u_{i} f_{i} ; \\
g_{2,1} & =y_{2}\left(u_{i} f_{i}-1\right) q_{1}, \quad g_{2,2}=(r-1) u_{i} f_{i}-q_{1}+1 ; \\
g_{3,1} & =\left(u_{i} f_{i}-1\right) y_{2}, \quad g_{3,2}=u_{i} f_{i}-1-g_{1}, \\
g_{3,3} & =y_{2}\left(u_{i} f_{i}-1\right) q_{1}-g_{2} ; \\
g_{4} & =q_{1} .
\end{aligned}
$$

Note that the appearance of $g_{1,1}$ or $g_{2,1}$ implies that $g_{3}=0$. Furthermore, $g_{1, i}+g_{3,2}=g_{1,1}$ and $g_{2, j}+g_{3,3}=g_{2,1}$. Finally, it is easily to verify that $g_{2}=g_{2,2}$ implies $g_{3,1}>g_{3,2}$. Thus, there are only three distinct expressions for $g$, namely

$$
\begin{aligned}
& g_{1,1}+g_{2,1}+g_{4}, \\
& g_{1,1}+g_{2,2}+g_{4}=g_{1,2}+g_{2,2}+g_{3,2}+g_{4}, \\
& g_{1,2}+g_{2,1}+g_{4}=g_{1,2}+g_{2,2}+g_{3,3}+g_{4} .
\end{aligned}
$$

Let $h$ denote the number of required middle-stage switches. We consider the following three cases related to the expressions presented above.

CASE 1. $g_{1,1}+g_{2,1}+g_{4}$. In this case we can distinguish the following three kinds of connections.

(1) The connections $s_{1}-t_{1}$ to $O-S_{2}$ can fully occupy bundles of links coming to $\left[\left(u_{i} f_{i}-1\right) /\left(v f_{o}\right)\right\rfloor$ middle-stage switches.

(2) The connections $S_{2}-T_{2}$ to $I-\left\{S_{1} \cup T_{1}\right\}$ can fully occupy bundles of links coming to $y_{2}\left\lfloor q_{1}\left(u_{i} f_{i}-1\right) /\left(v f_{o}\right)\right\rfloor$ middle-stages switches.

(3) The remaining $q_{1}$ connections are established while the bundles of links connecting middle-stage switches and third-stage switches belonging to $S_{2}$ are partially occupied by $y_{2} R_{1}=y_{2}\left(q_{1}\left(u_{i} f_{i}-1\right)\right) \bmod \left(v f_{o}\right)$ connections $S_{2}-T_{2}$ to $I-\left\{s_{1} \cup T_{1}\right\}$. The connections $T_{1}$ to $T_{2}$ can be established by partially occupied bundles of links if $q_{1} \leq y_{2}\left(v f_{o}-R_{1}\right)$. These links lead to $\left\lceil q_{1} /\left(v f_{o}-R_{1}\right)\right\rceil=\left\lfloor\left(q_{1}-1\right) /\left(v f_{o}-R_{1}\right)\right\rfloor+1$ middle-stage switches. If $q_{1}>y_{2}\left(v f_{o}-R_{1}\right)$ then the connections $T_{1}$ to $T_{2}$ and the connections partially occupying bundles of links can be established through $\left\lceil\left(q_{1}+y_{2} R_{1}\right) /\left(v f_{o}\right)\right\rceil$ middle-stage switches.

In the worst state the sets of switches described in (1)-(3) are disjoint. Hence, the number of required middle-stage switches is

$$
h=\left\lfloor\left(u_{i} f_{i}-1\right) /\left(v f_{o}\right)\right\rfloor+y_{2}\left\lfloor\left(q_{1}\left(u_{i} f_{i}-1\right)\right) /\left(v f_{o}\right)\right\rfloor+d,
$$


where

$$
d=\left\{\begin{array}{l}
\left\lfloor\left(q_{1}-1\right) /\left(v f_{o}-R_{1}\right)\right\rfloor+1, \quad \text { for } q_{1} \leq y_{2}\left(v f_{o}-R_{1}\right), \\
\left\lfloor\left(q_{1}+y_{2} R_{1}-1\right) /\left(v f_{o}\right)\right\rfloor+1, \text { for } q_{1}>y_{2}\left(v f_{o}-R_{1}\right) .
\end{array}\right.
$$

CASE 2. $g_{1,1}+g_{2,2}+g_{4}=g_{1,2}+g_{2,2}+g_{3,2}+g_{4}$. In this case we can distinguish the following three kinds of connections.

(1) $v f_{o}\left\lfloor\left(u_{i} f_{i}-1\right) /\left(v f_{o}\right)\right\rfloor$ connections $s_{1}-t_{1}$ to $O-S_{2}$ fully occupy bundles of links coming to $\left[\left(u_{i} f_{i}-1\right) /\left(v f_{v}\right)\right\rfloor$ middle-stage switches.

(2) The connections $S_{2}-T_{2}$ to $I-\left\{s_{1} \cup T_{1}\right\}$ can fully occupy bundles of links coming to at most $y_{2}\left\lfloor q_{1}\left(u_{i} f_{i}-1\right) /\left(v f_{o}\right)\right\rfloor$ middle-stage switches. However, the set $I-\left\{s_{1} \cup T_{1}\right\}$ limits the number of these connections to $g_{2,2}=$ $u_{i} f_{i}(r-1)-q_{1}+1$. In the worst state, output channels belonging to $S_{2}-T_{2}$ are also connected to $R=\left(u_{i} f_{i}-1\right) \bmod \left(v f_{o}\right)$ input channels belonging to $s_{1}-t_{1}$ which have not been used in (1). Hence, the connections described here fully occupy bundles of links coming to the following number of middle-stage switches

$$
\min \left\{y_{2}\left\lfloor\left(q_{1}\left(u_{i} f_{i}-1\right)\right) /\left(v f_{o}\right)\right\rfloor,\left\lfloor\left(u_{i} f_{i}(r-1)-q_{1}+R+1\right) /\left(v f_{o}\right)\right\rfloor\right\} .
$$

(3) The connections $T_{1}$ to $T_{2}$ are established while the bundles of links connecting middle-stage switches and third-stage switches belonging to $S_{2}$ are partially occupied by the following number of connections described in (2)

$$
\begin{aligned}
R_{2}= & u_{i} f_{i}(r-1)-q_{1}+1+R \\
& -\min \left\{y_{2}\left\lfloor\left(q_{1}\left(u_{i} f_{i}-1\right)\right) /\left(v f_{o}\right)\right\rfloor,\left\lfloor\left(u_{i} f_{i}(r-1)-q_{1}+R+1\right) /\left(v f_{o}\right)\right\rfloor\right\} v f_{o} .
\end{aligned}
$$

If $R_{1}=0$ or

$$
\left\lfloor\left(u_{i} f_{i}(r-1)-q_{1}+R_{1}+1\right) /\left(v f_{o}\right)\right\rfloor<y_{2}\left\lfloor\left(q_{1}\left(u_{i} f_{i}-1\right)\right) /\left(v f_{o}\right)\right\rfloor
$$

then, in the worst state, these remaining connections are established to a single switch belonging to $S_{2}$. In this case the connections $T_{1}$ to $T_{2}$ require $\left\lceil\left(q_{1}+R_{2}\right) /\left(v f_{o}\right)\right\rceil=\left\lfloor\left(q_{1}+R_{2}-1\right) /\left(v f_{o}\right)\right\rfloor+1$ middle-stage switches.

If $R_{1} \neq 0$ and

$$
\left\lfloor\left(u_{i} f_{i}(r-1)-q_{1}-R_{1}+1\right) /\left(v f_{o}\right)\right\rfloor \geq y_{2}\left\lfloor\left(q_{1}\left(u_{i} f_{i}-1\right)\right) /\left(v f_{o}\right)\right\rfloor
$$

then, in the worst state, these remaining connections are established to $\left\lceil R_{2} / R_{1}\right\rceil$ switches belonging to $S_{2}$. In this case, similarly as in Case 1 , the connections $T_{1}$ and $T_{2}$ require $\left\lfloor\left(q_{1}-1\right) /\left(v f_{0}-R_{1}\right)\right\rfloor+1$ middle-stage switches for $q_{1} \leq\left\lceil R_{2} / R_{1}\right\rceil v f_{o}-R_{2}$ and $\left\lfloor\left(q_{1}+R_{2}-1\right) /\left(v f_{o}\right)\right\rfloor+1$ middle-stage switches for $q_{1}>\left\lceil R_{2} / R_{1}\right\rceil v f_{o}-R_{2}$.

In the worst state sets of switches described in (1)-(3) are disjoint. Hence, the number of required middle-stage switches is

$$
\begin{aligned}
h=\left\lfloor\left(u_{i} f_{i}-1\right) /\left(v f_{o}\right)\right\rfloor+\min & \left\{y_{2}\left\lfloor\left(q_{1}\left(u_{i} f_{i}-1\right)\right) /\left(v f_{o}\right)\right\rfloor,\right. \\
& \left.\left\lfloor\left(u_{i} f_{i}(r-1)-q_{1}+R+1\right) /\left(v f_{o}\right)\right\rfloor\right\}+d_{1},
\end{aligned}
$$

where $d_{1}$ is expressed by (8). 
By using formula (4) we can rearrange formula (12) to

$$
\begin{array}{r}
h=\min \left\{\left\lfloor\left(u_{i} f_{i}-1\right) /\left(v f_{o}\right)\right\rfloor+y_{2}\left\lfloor\left(q_{1}\left(u_{i} f_{i}-1\right)\right) /\left(v f_{o}\right)\right\rfloor,\right. \\
\left.\left\lfloor\left(r u_{i} f_{i}-q_{1}\right) /\left(v f_{o}\right)\right\rfloor\right\}+d_{1} .
\end{array}
$$

Remark 1. We can note that $g_{1,2}<g_{1,1}$ only if $r=y_{2}$, i.e. $g_{1,2}=0$. Therefore, for $g_{1,2}+g_{2,2}+g_{3,2}+g_{4}$, (1) is related to connections $s_{1}-t_{1}$ to $S_{2}-T_{2}$, but not to connections $s_{1}-t_{1}$ to $0-S_{2}$.

REMARK 2. For $y_{2} q_{1}\left(u_{i} f_{i}-1\right) \leq u_{i} f_{i}(r-1)-q_{1}+R+1$ the number of required middle-stage switches for Case 2 is equal or greater than that for Case 1.

CASE 3. $g_{1,2}+g_{2,1}+g_{4}=g_{1,2}+g_{2,2}+g_{3,3}+g_{4}$. In this case we can distinguish the following three kinds of connections

(1) $v f_{o} y_{2}\left\lfloor\left(q_{1}\left(u_{i} f_{i}-1\right)\right) /\left(v f_{o}\right)\right\rfloor$ connections $S_{2}-T_{2}$ to $I-\left\{s_{1} \cup T_{1}\right\}$ fully occupy bundles of links coming to $y_{2}\left\lfloor\left(q_{1}\left(u_{i} f_{i}-1\right)\right) /\left(v f_{o}\right)\right\rfloor$ middle-stage switches.

(2) The set $O-S_{2}$ limits the number of the connections $s_{1}-t_{1}$ to $O-S_{2}$ to $u_{i} f_{i}\left(r-y_{2}\right)$. In case $g_{1,1}>g_{1,2}$, input channels belonging to $s_{1}-t_{1}$ can be connected to $\min \left\{u_{i} f_{i}-1, y_{2} R_{1}\right\}$ output channels belonging to $S_{2}-T_{2}$, because $y_{2} R_{1}$ channels belonging to $S_{2}-T_{2}$ have not been used in (1). Thus, the connections described here can fully occupy bundles of links coming to the following number of middle-stage switches

$$
\min \left\{\left\lfloor\left(u_{i} f_{i}-1\right) /\left(v f_{o}\right)\right\rfloor,\left\lfloor\left(\left(r-y_{2}\right) u_{i} f_{i}+y_{2} R_{1}\right) /\left(v f_{o}\right)\right\rfloor\right\} .
$$

(3) The output channels belonging to $S_{2}-T_{2}$ which have not been used in (1) and (2) can use the following number of connections with free channels belonging to $I-\left\{s_{1} \cup T_{1}\right\}$

$$
\begin{aligned}
R_{3}=\min & \left\{y_{2} R_{1},\left(r-y_{2}\right) u_{i} f_{i}+y_{2} R_{1}\right. \\
& \left.-\min \left\{\left\lfloor\left(u_{i} f_{i}-1\right) /\left(v f_{o}\right)\right\rfloor,\left\lfloor\left(\left(r-y_{2}\right) u_{i} f_{i}+y_{2} R_{1}\right) /\left(v f_{o}\right)\right\rfloor\right\} v f_{o}\right\} .
\end{aligned}
$$

In the worst state these connections are established with $\left\lceil R_{3} / R_{1}\right\rceil$ switches belonging to $S_{2}$, if $R_{1} \neq 0$. Similarly, as in Case 1 , the connections $T_{1}$ to $T_{2}$ require $\left\lfloor\left(q_{1}-1\right) /\left(v f_{o}-R_{1}\right)\right\rfloor+1$ middle-stage switches if $q_{1} \leq\left\lceil R_{3} / R_{1}\right\rceil v f_{o}-R_{3}$ and $\left\lfloor\left(q_{1}+R_{3}-1\right) /\left(v f_{o}\right)\right\rfloor+1$ middle-stage switches if $q_{1}>\left\lceil R_{3} / R_{1}\right\rceil v f_{o}-R_{3}$. If $R_{1}=0$, then $R_{3}=0$ and the connections $T_{1}$ and $T_{2}$ require $\left\lfloor\left(q_{1}-1\right) /\left(v f_{o}\right)\right\rfloor+1$ middle-stage switches.

In the worst state the sets of middle-stage switches described in (1)-(3) are disjoint. Hence, the number of required middle-stage switches is

$$
\begin{aligned}
h=y_{2}\left\lfloor\left(q_{1}\left(u_{i} f_{i}-1\right)\right) /\left(v f_{o}\right)\right\rfloor+ & \min \left\{\left\lfloor\left(u_{i} f_{i}-1\right) /\left(v f_{o}\right)\right\rfloor,\right. \\
& \left.\left\lfloor\left(\left(r-y_{2}\right) u_{i} f_{i}+y_{2} R_{1}\right) /\left(v f_{o}\right)\right\rfloor\right\}+d_{2},
\end{aligned}
$$

where $d_{2}$ is expressed by (9). 


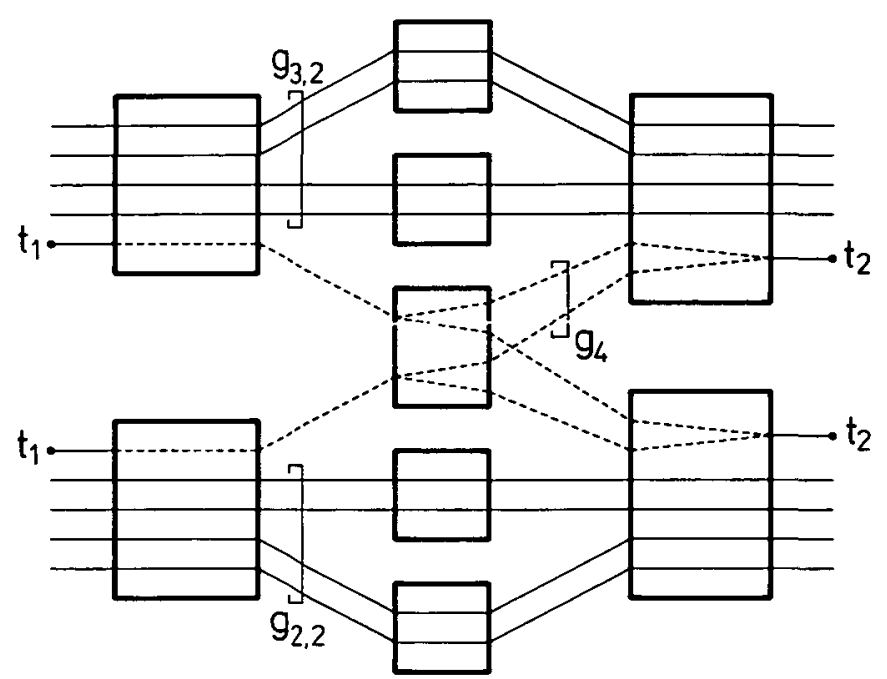

FiguRE 3. The worst state in the $(2,2)$-multiconnection network controlled by using Strategy 2.

By using formula (4) we can rewrite (14) obtaining the following expression

$$
\begin{aligned}
h=\min \left\{\left\lfloor\left(u_{i} f_{i}-1\right) /\left(v f_{o}\right)\right\rfloor+\right. & \left\lfloor\left(q_{1}\left(u_{i} f_{i}-1\right)\right) /\left(v f_{o}\right)\right\rfloor, \\
& \left.\left\lfloor\left(\left(r-y_{2}\right) u_{i} f_{i}-y_{2} q_{1}\left(u_{i} f_{i}-1\right)\right) /\left(v f_{o}\right)\right\rfloor\right\}+d_{2} .
\end{aligned}
$$

RemarK 3. For $u_{i} f_{i}-1 \leq\left(r-y_{2}\right) u_{i} f_{i}$ Case 3 is transformed to Case 1.

Summarising Cases 2 and 3 and choosing the maximum value of $h\left(q_{1}, y_{2}\right)$ for $1 \leq y_{2} \leq q_{2}$ we obtain Theorem 2 . Necessity can be proved with ease by constructing a sequence of calls which leads to the occupancy of the switches of formula (7).

Theorem 2 is illustrated by the following example.

EXAMPLE 2. Let us consider a network identical to that of Example 1. Under Strategy 2 this network is nonblocking for $m \geq 5$. The worst state of the network is illustrated in Figure 3. This state corresponds to Case 2 of the proof, and, in particular, to the combination: $g_{1,2}+g_{2,2}+g_{3,2}+g_{4}$, where $g_{1,2}=0$. Note that Case 2 is proved only for the combination $g_{1,1}+g_{2,2}+g_{4}$ which gives the same $m$ as the combination appearing in our example.

THEOREM 3. A two-sided three-stage network composed of DSMs is nonblocking as a $\left(q_{1}, q_{2}\right)$-multiconnection network under Strategy 3 if and only if

$$
\begin{aligned}
& m \geq \max _{1 \leq y_{1} \leq q_{1}}\left\{\operatorname { m i n } \left\{\left\lfloor\left(u_{i} f_{i}-1\right) /\left(v f_{o}\right)\right\rfloor+y_{1}\left\lfloor\left(q_{2}\left(u_{i} f_{i}-1\right)\right) /\left(v f_{o}\right)\right\rfloor\right.\right. \\
&+ \min \left\{d_{3}, d_{4}\right\},\left\lfloor\left(r u_{i} f_{i}-q_{2}\right) /\left(v f_{o}\right)\right\rfloor \\
&+\left.\left.d_{3},\left\lfloor\left(\left(r-y_{1}\right) u_{i} f_{i}+q_{2} y_{1}\left(u_{i} f_{i}-1\right)\right) /\left(v f_{o}\right)\right\rfloor+d_{4}\right\}\right\},
\end{aligned}
$$


where

$$
\begin{aligned}
& d_{3}=\left\{\begin{array}{c}
\left\lfloor\left(q_{2}-1\right) /\left(v f_{o}-R_{1}\right)\right\rfloor+1, \text { for } q_{2} \leq\left\lceil R_{2} / R_{1}\right\rceil v f_{o}-R_{2}, R_{1} \neq 0, \text { and } \\
y_{1}\left\lfloor\left(q_{2}\left(u_{i} f_{1}-1\right)\right) /\left(v f_{o}\right)\right\rfloor \leq\left\lfloor\left(u_{i} f_{i}(r-1)-q_{2}+R+1\right) /\left(v f_{o}\right)\right\rfloor \\
\left\lfloor\left(q_{2}+R_{2}-1\right) /\left(v f_{o}\right)\right\rfloor+1, \text { for } q_{2}>\left\lceil R_{2} / R_{1}\right\rceil v f_{o}-R_{2}, R_{1} \neq 0 \\
\quad \text { or } y_{1}\left\lfloor\left(q_{2}\left(u_{i} f_{i}-1\right)\right) /\left(v f_{o}\right)\right\rfloor>\left\lfloor\left(u_{i} f_{i}(r-1)-q_{2}+R+1\right) /\left(v f_{o}\right)\right\rfloor \\
\quad \text { or } R_{1}=0
\end{array}\right. \\
& d_{4}=\left\{\begin{array}{l}
\left\lfloor\left(q_{2}-1\right) /\left(v f_{o}-R_{1}\right)\right\rfloor+1, \text { for } q_{2} \leq\left\lceil R_{3} / R_{1}\right\rceil v f_{o}-R_{3}, R_{1} \neq 0 \\
\left\lfloor\left(q_{2}+R_{3}-1\right) /\left(v f_{o}\right)\right\rfloor+1, \text { for } q_{2}>\left\lceil R_{3} / R_{1}\right\rceil v f_{o}-R_{3}, R_{1} \neq 0 \\
\left\lfloor\left(q_{2}-1\right) /\left(v f_{o}\right)\right\rfloor+1, \text { for } R_{1}=0
\end{array}\right. \\
& R=\left(u_{i} f_{i}-1\right) \bmod \left(v f_{o}\right) \text {; } \\
& R_{1}=\left(q_{2}\left(u_{i} f_{i}-1\right)\right) \bmod \left(v f_{o}\right) \text {; } \\
& R_{2}=u_{i} f_{i}(r-1)-q_{2}+1+R- \\
& -\min \left\{y_{1}\left\lfloor\left(q_{2}\left(u_{i} f_{i}-1\right)\right) /\left(v f_{o}\right)\right\rfloor,\left\lfloor\left(u_{i} f_{i}(r-1)-q_{2}+R+1\right) /\left(v f_{o}\right)\right\rfloor\right\} v f_{o} ; \\
& R_{3}=\min \left\{y_{1} R_{1},\left(r-y_{1}\right) u_{i} f_{i}+y_{1} R_{1}-\right. \\
& \left.-\min \left\{\left\lfloor\left(u_{i} f_{i}-1\right) /\left(v f_{o}\right)\right\rfloor,\left\lfloor\left(\left(r-y_{1}\right) u_{i} f_{i}+y_{1} R_{1}\right) /\left(v f_{o}\right)\right\rfloor\right\} v f_{o}\right\} \text {. }
\end{aligned}
$$

Proof. Analogous to the proof of Theorem 2

THEOREM 4. A two-sided three-stage network composed of DSMs is nonblocking as a $\left(q_{1}, q_{2}\right)$-multiconnection network under Strategy 4 if and only if

$$
\begin{aligned}
m \geq 1+\max _{\substack{1 \leq y_{1} \leq q_{1} \\
1 \leq y_{2} \leq q_{2}}}\left\{\operatorname { m i n } \left\{\left(y_{1}+y_{2}\right)\left\lfloor\left(u_{i} f_{i}-1\right) /\left(v f_{o}\right)\right\rfloor,\right.\right. \\
\\
\left.\left.\quad\left\lfloor\left(r u_{i} f_{i}-\max \left\{y_{1}, y_{2}\right\}\right) /\left(v f_{o}\right)\right\rfloor\right\}\right\} .
\end{aligned}
$$

Proof. Sufficiency. Consider the connection of the pair $\left(T_{1}, T_{2}\right)$. Let $g_{1}$ be the maximum number of connections between the sets $S_{1}-T_{1}$ and $O-S_{2}$. Let $g_{2}$ be the maximum number of connections between $S_{2}-T_{2}$ and $I-S_{1}$. Let $g_{3}$ be the maximum number of connections between $S_{1}-T_{1}$ and $S_{2}-T_{2}$. Let $g_{4}$ be the number of connections between $T_{1}$ and $T_{2}$.

The numbers $g_{1}$ to $g_{4}$, for the worst state of a network, are as follows:

$$
\begin{aligned}
& g_{1}=\min \left\{y_{1}\left(u_{i} f_{i}-1\right),\left(r-y_{2}\right) u_{i} f_{i}\right\}, \\
& g_{2}=\min \left\{y_{2}\left(u_{i} f_{i}-1\right),\left(r-y_{1}\right) u_{i} f_{i}\right\}, \\
& g_{3}=\min \left\{y_{1}\left(u_{i} f_{i}-1\right)-g_{1}, y_{2}\left(u_{i} f_{i}-1\right)-g_{2}\right\}, \\
& g_{4}=1 .
\end{aligned}
$$

The total number of connections is then $g=g_{1}+g_{2}+g_{3}+g_{4}$. 
Similarly as in the proof of Theorem 2 we consider all possible combinations of the terms $g_{1}, g_{2}, g_{3}$, and $g_{4}$. Write

$$
\begin{aligned}
& g_{1,1}=y_{1}\left(u_{i} f_{i}-1\right), \quad g_{1,2}=\left(r-y_{2}\right) u_{i} f_{i} ; \\
& g_{2,1}=y_{2}\left(u_{i} f_{i}-1\right), \quad g_{2,2}=\left(r-y_{1}\right) u_{i} f_{i} ; \\
& g_{3,1}=y_{1}\left(u_{i} f_{i}-1\right)-g_{1}, \quad g_{3,2}=y_{2}\left(u_{i} f_{i}-1\right)-g_{2} .
\end{aligned}
$$

Note that the appearance of $g_{1,1}$ or $g_{2,1}$ implies that $g_{3}=0$.

Furthermore, $g_{1, i}+g_{3,1}=g_{1,1}$ and $g_{2, j}+g_{3,2}=g_{2,1}$. Thus, it is sufficient to consider the three combinations

$$
\begin{aligned}
& g_{1,1}+g_{2,1}+g_{4}, \\
& g_{1,1}+g_{2,2}+g_{4}=g_{1,2}+g_{2,2}+g_{3,1}+g_{4}, \\
& g_{1,2}+g_{2,1}+g_{4}=g_{1,2}+g_{2,2}+g_{3,2}+g_{4}
\end{aligned}
$$

Let $h$ denote the number of required middle-stage switches. We consider the following three cases related to the expressions presented above.

CASE 1. $g_{1,1}+g_{2,1}+g_{4}$. In this case we can distinguish the following two kinds of connections.

(1) Between the sets $S_{1}-T_{1}$ and $O-S_{2} y_{1}\left(u_{i} f_{i}-1\right)$ connections are established. These connections can fully occupy bundles of links coming to $y_{1}\left\lfloor\left(u_{i} f_{i}-1\right) /\left(v f_{o}\right)\right\rfloor$ middle-stage switches.

(2) Between the sets $S_{2}-T_{2}$ and $I-S_{1} y_{2}\left(u_{i} f_{i}-1\right)$ connections are established. These connections can fully occupy bundles of links coming to $y_{2}\left\lfloor\left(u_{i} f_{i}-1\right) /\left(v f_{o}\right)\right\rfloor$ middle-stage switches.

In the worst state the sets of switches described in (1) and (2) are disjoint. Because in order to establish the connection $T_{1}$ to $T_{2}$ an additional switch is required, the total number of middle-stage switches is

$$
h=\left(y_{1}+y_{2}\right)\left\lfloor\left(u_{i} f_{i}-1\right) /\left(v f_{o}\right)\right\rfloor .
$$

CASE 2. $g_{1,1}+g_{2,2}+g_{4}=g_{1,2}+g_{2,2}+g_{3,1}+g_{4}$. In this case we can distinguish the following two kinds of connections

(1) The set $S_{1}-T_{1}$ can realise $y_{1}\left(u_{i} f_{i}-1\right)$ connections; $v f_{o} y_{1}\left\lfloor\left(u_{i} f_{i}-\right.\right.$ 1)/ $\left.\left(v f_{0}\right)\right\rfloor$ of them can fully occupy bundles of links coming to $y_{1}\left\lfloor\left(u_{i} f_{i}-\right.\right.$ 1) $\left./\left(v f_{o}\right)\right\rfloor$ middle-stage switches.

(2) Theoretically, the connections realised by set $S_{2}-T_{2}$ can fully occupy bundles of links coming to at most $y_{2}\left\lfloor\left(u_{i} f_{i}-1\right) /\left(v f_{o}\right)\right\rfloor$ middle-stage switches. However, the set $I-S_{1}$ limits the number of connections between $S_{2}-T_{2}$ and $I-S_{1}$ to $\left(r-y_{1}\right) u_{i} f_{l}$. The output channels belonging to $S_{2}-T_{2}$ can be additionally connected to $y_{1} R=y_{1}\left(u_{i} f_{i}-1\right) \bmod \left(v f_{o}\right)$ input channels belonging to $S_{1}-T_{1}$, which have not been used in (1). Therefore, the 


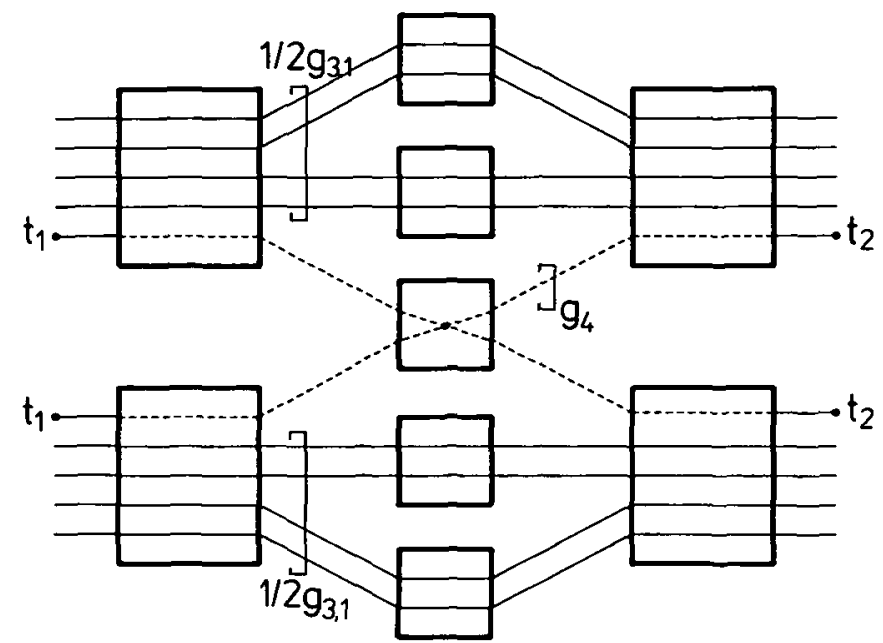

FIGURE 4. The worst state in the $(2,2)$-multiconnection network controlled by using Strategy 4.

connections described in (2) can fully occupy bundles of links coming to $\min \left\{\left\lfloor\left(\left(r-y_{1}\right) u_{i} f_{i}+y_{1} R\right) /\left(v f_{o}\right)\right\rfloor, y_{2}\left\lfloor\left(u_{i} f_{i}-1\right) /\left(v f_{o}\right)\right\rfloor\right\}$ middle-stage switches.

In the worst state the sets of switches described in (1) and (2) are disjoint. Because in order to establish the connection $T_{1}$ to $T_{2}$ an additional switch is required, the total number of middle-stage switches is

$$
\begin{aligned}
h=y_{1}\left\lfloor\left(u_{i} f_{i}-1\right) /\left(v f_{o}\right)\right\rfloor+\min \left\{y_{2}\left\lfloor\left(u_{i} f_{i}-1\right) /\left(v f_{o}\right)\right\rfloor,\right. \\
\\
\left.\left\lfloor\left(\left(r-y_{1}\right) u_{i} f_{i}+y_{1} R\right) /\left(v f_{o}\right)\right\rfloor\right\}+1 .
\end{aligned}
$$

By using formula (4) we can rewrite (21) obtaining the following expression

$$
h=1+\min \left\{\left(y_{1}+y_{2}\right)\left\lfloor\left(u_{i} f_{i}-1\right) /\left(v f_{o}\right)\right\rfloor,\left\lfloor\left(r u_{i} f_{i}-y_{1}\right) /\left(v f_{o}\right)\right\rfloor\right\} \text {. }
$$

CASE 3. $g_{1,2}+g_{2,1}+g_{4}=g_{1,2}+g_{2,2}+g_{3,2}+g_{4}$. By symmetry to Case 2 we have

$$
h=1+\min \left\{\left(y_{1}+y_{2}\right)\left\lfloor\left(u_{i} f_{i}-1\right) /\left(v f_{o}\right)\right\rfloor,\left\lfloor\left(r u_{i} f_{i}-y_{2}\right) /\left(v f_{o}\right)\right\rfloor\right\} .
$$

Summarising Cases 1 to 3 and choosing the maximum value of $h\left(y_{1}, y_{2}\right)$ for $1 \leq y_{1} \leq q_{1}$ and $1 \leq y_{2} \leq q_{2}$ we obtain Theorem 4 . Necessity can be proved by constructing a sequence of calls which leads to the occupancy of the switches of formula (19).

EXAMPLE 3. Let us consider a network identical to that of Example 1. Under Strategy 4 this network is nonblocking for $m \geq 5$. The worst state of the network is illustrated in Figure 4. This state corresponds to the combination: $g_{1,2}+g_{2,2}+g_{3,1}+g_{4}$, where $g_{1,2}=g_{2,2}=0, g_{3,1}=8$, and $g_{4}=1$. 
We note that for $f_{i}=f_{o}=v=1$ Theorems $1,2,3$, and 4 all reduce to the theorems presented in [5], for the symmetrical Clos network. For $q_{1}=q_{2}=1$, for all theorems, we obtain the known result: $m \geq 2\left\lfloor\left(u_{i} f_{i}-1\right) /\left(v f_{o}\right)\right\rfloor+1$ [9]. For $r>q_{1} q_{2}$ Theorems 1 to 4 reduce to the theorems presented in [10]. For $f_{i}=f_{o}=v=q_{1}=q_{2}=1$ we obtain the well-known Clos theorem [3].

\section{Conclusion}

In the paper the conditions under which three-stage symmetrical networks composed of digital switching matrices are nonblocking as $\left(q_{1}, q_{2}\right)$-multiconnection networks were studied. We can note that although our results have been developed for time-division networks, they are valid also for multiple linkage space-division networks, i.e. networks in which each pair of switches belonging to adjacent stages is connected by more than one link. The obtained results can be easily extended also for non-symmetrical networks, as well as for networks containing more than three-stages.

Since nonblocking multiconnection networks require expansion in the first stage and concentration in the last one we can form various expansion and concentration modules made of square switches (available DSMs are usually square) by using the method proposed in [8]. In some practical applications other approaches to multiconnections may be justified [11], [15].

Throughout the paper we assumed that each rectangular switch has the fan-in and fan-out properties. However, in most cases digital time-division switches have only the fan-out property, i.e. any input channel can be connected to a multiplicity of output channels but not vice versa. In this case Strategies 1 and 3 and Strategies 2 and 4 are in pairs equivalent and, therefore, Theorem 1 is identical to Theorem 3 , and Theorem 2 is identical to Theorem 4. The fan-out property does not limit the construction of broadcast networks, i.e. $(1, q)$-multiconnection networks, which are of practical interest, especially in broadband applications.

\section{References}

[1] P. Belforte, B. Bostica, V. Masina, and L. Pilati, “Design and development of an LSI digital switching element (ECI)", CSELT Rapporti tecnici 13 (1984) 115-123.

[2] P. Charransol, J. Hauri, C. Athènes, and D. Hardy, "Development of a time division switching network usable in a very large range of capacities ", IEEE Trans. Commun., COM-27 (1979) 982-988. 
[3] C. Clos, "A study of non-blocking switching networks", Bell System Tech. J. 32 (1953) 406-424.

[4] F. K. Hwang, "Three-stage multiconnection networks which are nonblocking in the wide sense", Bell System Tech. J. 58 (1979) 2183-2187.

[5] F. K. Hwang and A. Jajszczyk, “On nonblocking multiconnection networks”, IEEE Trans. Commun., COM-34 (1986) 1038-1041.

[6] A. Jajszczyk, "Comments on three-stage multiconnection networks which are nonblocking in the wide sense", Bell System Tech. J. 62 (1983) 2113-2114.

[7] A. Jajszczyk, "Design of switching networks composed of uniform time space elements", Proc. IIth Int. Teletraffic Congress, (North-Holland, Amsterdam 1985) 1061-1068.

[8] A. Jajszczyk, "Novel architecture for a digital switching network", Electron. Lett. 20 (1984) 683.

[9] A. Jajszczyk, “On nonblocking switching networks composed of digital symmetrical matrices", IEEE Trans. Commun., COM-31 (1983) 2-9.

[10] A. Jajszczyk, Switching networks composed of time-space switches (Technical University of Poznań Press, Poznañ, 1985), in Polish.

[11] A. Jajszczyk and W. Kabaciński, "Methods for the connection of signalling units to switching networks", Electron. Lett. 23 (1987) 443-444.

[12] J. C. McDonald (ed.), Fundamentals of digital switching (Plenum Press, New York, 1983).

[13] Y. Shimazu and T. Tanaka, "High-speed time switch using GaAs LSI technology', IEEE J. Select. Areas Commun., SAC-4 (1986) 32-38.

[14] D. R. Spears, "Broadband ISDN switching capabilities from a service perspective", IEEE J. Select. Areas Commun., SAC-5 (1987) 1222-1230.

[15] H. Yamada, H. Kataoka, T.Sampei, and T. Yano, "High-speed digital switching technology using space-division-switch LSI's", IEEE J. Select. Areas Commun., SAC-4 (1986) 529-535.

[16] N. Yamanaka, H. Miyanaga, and Y. Yamamoto, "High-speed time division switch for 32-Mbit/s bearer rate signals", IEEE J. Select. Areas Commun., SAC-5 (1987) 1249-1255. 